\title{
Gesundheitsausgaben: Schweiz auf dem zweiten und dritten Rang
}

\author{
Gemessen am Anteil am Bruttoinlandsprodukt hat die Schweiz wiederum die \\ zweithöchsten Gesundheitsausgaben der Welt (2003). Bezüglich Pro-Kopf- \\ Ausgaben ist die Schweiz nach den USA und Norwegen auf dem dritten Rang. \\ Extrem hoch sind in unserem Land die Barauslagen der Haushalte.
}

G. Kocher

Korrespondenz:

Dr. rer. pol. Gerhard Kocher

Haldenweg 10a

CH-3074 Muri b. Bern

Tel. 0319526707

kocher@spectraweb.ch
Die OECD in Paris hat die neueste jährliche Übersicht über die Gesundheitswesen der 30 OECDLänder als CD-ROM [1] und gekürzt in Buchform [2] publiziert. Die OECD ist mit der WHO weltweit die führende Quelle für Vergleiche der Gesundheitssysteme der Industrieländer.

\section{Anteil der Gesundheitskosten am BIP}

Die Tabelle 1 zeigt in der zweiten und dritten Spalte die Anteile am Bruttoinlandsprodukt BIP. Wie immer sind die USA mit weitem Vorsprung auf dem ersten Rang: Ihr Anteil (15\%) ist nicht weniger als $72 \%$ höher als der OECD-Durchschnitt und 30\% höher als das zweitplazierte Land, die Schweiz [3]. Wie im Vorjahr 2002 folgt auf dem dritten Rang Deutschland mit 11,1\%. Es folgen Island, Norwegen und Frankreich. Die Schweiz ist seit 1999 auf dem zweiten Rang (teuerstes Gesundheitswesen Europas). Der Anteil der Schweiz $(11,5 \%)$ ist wie im Vorjahr ein Drittel höher als der OECD-Durchschnitt. Unter dem Durchschnitt liegen unter anderem Italien, Grossbritannien, Österreich und Finnland.

Im Vergleich zu 2002 konnten drei der 30 Länder ihren Gesundheitskostenanteil am BIP leicht senken (Luxemburg, Neuseeland und Polen). In Österreich und Italien blieb der Anteil gleich. In den OECD-Ländern insgesamt stieg der Anteil zwischen 2002 und 2003 von 8,6 auf 8,7\%. Bei diesen Vergleichen ist $\mathrm{zu}$ beachten, dass der Anteil der Gesundheitsausgaben am Bruttoinlandsprodukt definitionsgemäss nicht nur von der Kostenentwicklung im Gesundheitssystem abhängt, sondern auch von der wirtschaftlichen Entwicklung des Landes insgesamt.

Wie veränderte sich der Anteil am BIP zwischen 2000 und 2003? Gesamthaft stieg er um 9,9\%, am stärksten in Norwegen (um 21,2\%), überdurchschnittlich in den USA (um 14,5\%) und auch knapp in der Schweiz (um 10,6\%). Nur leicht war das Wachstum in Deutschland $(4,7 \%)$, ein Nullwachstum verzeichnete Österreich.

\section{Pro-Kopf-Ausgaben}

Die zweite wichtige Vergleichsgrösse sind die Pro-Kopf-Ausgaben für das Gesundheitswesen (Spalten 4 und 5). Diese Dollarbeträge sind kaufkraftbereinigt, berücksichtigen also die unterschiedliche Kaufkraft in den einzelnen Ländern. Wiederum abgehoben sind hier die USA mit \$ 5635, gefolgt von Norwegen mit \$ 3807 und der Schweiz mit \$3781. Gegenüber 2002 ist hier die Schweiz um einen Rang zurückgefallen; seit 1990 war sie immer auf dem zweiten Rang. Der OECD-Durchschnitt beträgt \$ 2340 (Vorjahr $\$ 2246)$. Die USA gaben im Vergleich zum Durchschnitt 141\% mehr aus, die Schweiz 62\%. Die einsame Spitzenposition der USA zeigt sich auch darin, dass sie $48 \%$ mehr ausgaben als das zweitplazierte Land (Norwegen).

Der Vergleich 2003 mit dem Jahr 2000 zeigt ein durchschnittliches Wachstum um 20,5\% (USA 24,1\%, Schweiz 18,8\%, Deutschland $12,2 \%)$. Den weitaus geringsten Anstieg (6,5\%) aller 30 Länder konnte Österreich verzeichnen.

\section{Starke Direktbelastung in der Schweiz}

Wie bereits im früheren Artikel [3] sticht auch in den neuesten OECD-Zahlen die ausserordentlich hohe (und noch steigende) Selbstzahlerbelastung in der Schweiz hervor. Die Bevölkerung zahlt 31,5\% der nationalen Gesundheitsausgaben direkt aus der eigenen Tasche (Out-of-pocketZahlungen), vor allem für rezeptfreie Medikamente, die Zahnmedizin, Pflegeheime und Spitex. Selbst im noch stärker privatwirtschaftlich orientierten US-Gesundheitswesen beträgt die Selbstzahlerbelastung nur 14,1\%, ist also fast dreimal kleiner. Nur für 18 der 30 Länder liegen die Prozentsätze vor. Im Schnitt dieser Staaten zahlt die Bevölkerung 20,8\% der nationalen Gesundheitsausgaben direkt aus eigener Tasche.

Die Schweiz ist in dieser Hinsicht ein Sonderfall: Nicht nur ist der Selbstzahleranteil extrem hoch, sondern er wird laufend noch erhöht 
(Franchise, Selbstbehalt). Dies, obwohl die Höhe der Gesamtausgaben in unserem Land - wir sind Europameister bezüglich Anteil am BIP und Europazweiter bezüglich Pro-Kopf-Ausgaben den Spareffekt dieser Finanzierungspolitik nicht gerade belegt...

2003 beliefen sich die Out-of-pocket-Zahlungen in der Schweiz pro Kopf auf \$ 1192 (kaufkraftbereinigt). Das sind 4\% mehr als 2002 (\$1149). Es folgten Griechenland mit \$ 935, die USA mit \$ 793 und Norwegen mit \$ 591. In Italien betrugen die Barauslagen $\$ 468$, in Deutschland \$ 312 und in Frankreich \$ 291.

\section{Internationaler Vergleich der Ärztedichte 2003}

Die Tabelle 2 zeigt die Zahl der berufstätigen Ärzte pro 1000 Einwohner. Im OECD-Durchschnitt sind es 2,9; die Extreme sind Griechenland (2001) mit 4,4 und die Türkei mit 1,4. Mit ihrer Ärztedichte $(3,6)$ liegt die Schweiz auf dem vierten Rang nach Griechenland, Italien und Belgien. Österreich hat Rang 7 zusammen mit Deutschland und Frankreich $(3,4)$. Die USA hatten 2002 eine Ärztedichte von 2,3, gefolgt von Neuseeland und Grossbritannien mit je 2,2 und Japan (2002) mit 2 Ärzten pro 1000 Einwohner. Die Schweizer Ärztedichte (2002) ist 24\% höher als der OECD-Durchschnitt.

\section{Prävention und öffentliche Gesundheits-}

\section{programme}

Die WHO kritisiert die geringen Ausgaben für Prävention und staatliche Gesundheitsprogramme. In den OECD-Ländern sind es durchschnittlich nur 2,9\% der nationalen Gesundheitsausgaben. Spitzenreiter sind die Niederlande mit 5,5\% (Kanada gibt $8 \%$ aus, schliesst aber die Verwaltungskosten aller Ministerien ein), gefolgt von Ungarn mit 5, Deutschland mit 4,8, den USA mit 3,9 und Finnland mit 3,8\%. Die Schweiz ist mit 2,2\% Präventionsausgaben auf dem 13 . von 21 Rängen. Schlusslicht ist Italien mit 0,6\%.

\section{Literatur}

1 Die CD-ROM «OECD Health Data 2005» kostet $€ 325$ (nichtkommerzielle Organisationen $€ 225$ ) und ist sechssprachig englisch/französisch/deutsch/ italienisch/spanisch/russisch (ISBN 92-64-00876-4). Unter www.oecd.org können Informationsmaterial sowie eine kostenlose Demoversion abgerufen werden.

2 Die wichtigsten Daten aus der erwähnten CD-ROM sind in einem 172seitigen Buch erschienen: «Panorama de la santé», OECD Paris, 2005, € 25 (ISBN 92-64-01262-1).

Englische Ausgabe: «Health at a Glance».

3 Kocher G. Zweitteuerstes Gesundheitswesen der Welt. Schweiz Ärztezeitung 2004;85(40):2142-4.

Tabelle 1

Gesundheitsausgaben 2003 in Prozent des Bruttoinlandprodukts BIP und in \$ (kaufkraftbereinigt) pro Kopf.

\begin{tabular}{|c|c|c|c|c|c|c|c|c|c|}
\hline Land & $\%$ BIP & Rang & $\$$ & Rang & Land & $\%$ BIP & Rang & $\$$ & Rang \\
\hline USA & 15 & 1 & 5635 & 1 & Neuseeland & 8,1 & 14 & 1886 & 21 \\
\hline Schweiz $^{b}$ & 11,5 & 2 & 3781 & 3 & Japan a,b & 7,9 & 15 & 2139 & 18 \\
\hline Deutschland & 11,1 & 3 & 2996 & 7 & Grossbritannien $^{a}$ & 7,7 & 16 & 2231 & 17 \\
\hline Island & 10,5 & 4 & 3115 & 5 & Spanien & 7,7 & 16 & 1835 & 22 \\
\hline Norwegen ${ }^{b}$ & 10,3 & 5 & 3807 & 2 & Österreich & 7,5 & 17 & 2302 & 15 \\
\hline Frankreich $^{\mathrm{b}}$ & 10,1 & 6 & 2903 & 9 & Tschechische Republik & 7,5 & 17 & 1298 & 24 \\
\hline$K a n a d a{ }^{b}$ & 9,9 & 7 & 3001 & 6 & Irland & 7,4 & 18 & 2451 & 14 \\
\hline Griechenland & 9,9 & 7 & 2011 & 20 & Finnland & 7,4 & 18 & 2118 & 19 \\
\hline Niederlande & 9,8 & 8 & 2976 & 8 & Türkei b & 7,4 & 18 & 513 & 30 \\
\hline Belgien & 9,6 & 9 & 2827 & 10 & Luxemburg & 6,9 & 19 & 3705 & 4 \\
\hline Portugal & 9,6 & 9 & 1797 & 23 & Polen & 6,5 & 20 & 744 & 28 \\
\hline Schweden & 9,4 & 10 & 2703 & 12 & Mexiko & 6,2 & 21 & 583 & 29 \\
\hline Australien & 9,3 & 11 & 2699 & 13 & Slowakei & 5,9 & 22 & 777 & 27 \\
\hline Dänemark & 9 & 12 & 2763 & 11 & Südkorea ${ }^{a}$ & 5,6 & 23 & 1074 & 26 \\
\hline Italien & 8,4 & 13 & 2258 & 16 & & & & & \\
\hline Ungarn ${ }^{b}$ & 8,4 & 13 & 1269 & 25 & Durchschnitt & 8,7 & & 2340 & \\
\hline
\end{tabular}


Tabelle 2

Ärztedichte 2003 und 2000. Berufstätige Ärzte je 1000 Einwohner.

\begin{tabular}{|c|c|c|c|}
\hline Länder & 2003 & 2000 & $\begin{array}{l}\text { Zu- oder } \\
\text { Abnahme in \% }\end{array}$ \\
\hline Griechenland $^{\mathrm{b}}$ & 4,4 & 4,3 & 2,3 \\
\hline Italien & 4,1 & 4,2 & $-2,4$ \\
\hline Belgien ${ }^{a, d}$ & 3,9 & 3,9 & 0,0 \\
\hline Island d & 3,6 & 3,4 & 5,9 \\
\hline Schweiz ${ }^{a}$ & 3,6 & 3,5 & 2,9 \\
\hline Tschechische Republike & 3,5 & 3,4 & 2,9 \\
\hline Österreich & 3,4 & 3,2 & 6,3 \\
\hline Deutschland & 3,4 & 3,3 & 3,0 \\
\hline Frankreich $^{d}$ & 3,4 & 3,3 & 3,0 \\
\hline Schweden ${ }^{a}$ & 3,3 & 3,1 & 6,5 \\
\hline Portugal & 3,3 & 3,2 & 3,1 \\
\hline Ungarnc ${ }^{c}$ & 3,2 & 3,1 & 3,2 \\
\hline Spanien & 3,2 & 3,2 & 0,0 \\
\hline Norwegen ${ }^{e}$ & 3,1 & 2,9 & 6,9 \\
\hline Niederlande $^{f}$ & 3,1 & 3,2 & $-3,1$ \\
\hline Slowakei & 3,1 & 3,2 & $-3,1$ \\
\hline
\end{tabular}

\begin{tabular}{|c|c|c|c|}
\hline Länder & 2003 & 2000 & $\begin{array}{l}\text { Zu- oder } \\
\text { Abnahme in \% }\end{array}$ \\
\hline Dänemarka & 2,9 & 2,8 & 3,6 \\
\hline Luxemburg $^{d}$ & 2,7 & 2,5 & 8,0 \\
\hline Irland $^{f}$ & 2,6 & 2,2 & 18,2 \\
\hline Finnland & 2,6 & 2,6 & 0,0 \\
\hline Polen & 2,5 & 2,2 & 13,6 \\
\hline Australien ${ }^{a}$ & 2,5 & 2,5 & 0,0 \\
\hline USA ${ }^{a, d}$ & 2,3 & 2,2 & 4,5 \\
\hline Grossbritannien & 2,2 & 1,9 & 15,8 \\
\hline Neuseeland & 2,2 & 2,2 & 0,0 \\
\hline Kanada & 2,1 & 2,1 & 0,0 \\
\hline Japana & 2 & 1,9 & 5,3 \\
\hline Südkorea & 1,6 & 1,3 & 23,1 \\
\hline Mexiko & 1,5 & 1,6 & $-6,3$ \\
\hline Türkei & 1,4 & 1,3 & 7,7 \\
\hline $\begin{array}{l}\text { Durchschnitt } \\
30 \text { Länder }\end{array}$ & 2,9 & 2,8 & 4,4 \\
\hline
\end{tabular}

a Spalte 2003: Zahl für 2002; b Spalte 2003: Zahl für 2001; c Spalte 2000: Zahl für 1999; d inkl. Ärzte in Industrie,

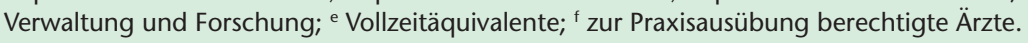

